Pacific Journal of Mathematics

POWERS OF IDEALS IN LOCALLY UNMIXED NOETHERIAN 


\title{
POWERS OF IDEALS \\ IN LOCALLY UNMIXED NOETHERIAN RINGS
}

\author{
L. J. RATLIFF, JR.
}

\begin{abstract}
It is known that the following statements are equivalent for a semi-local ring $R$ : (1) $R$ is analytically unramified; (2) There exists an open ideal $I$ in $R$ and an integer $n \geq 0$ such that $\left(I^{n+i}\right)_{a} \subseteq I^{i}$ for all $i \geq 1$, where $\left(I^{n+t}\right)_{a}$ is the integral closure of $I^{n+t}$. Moreover, if $R$ is analytically unramified and $I$ is any ideal in $R$, then (2) holds for $I$ and, (3) There exists an integer $m \geq 1$ such that, with $B=\left(I^{m}\right)_{a},\left(B^{i}\right)_{a}=B^{i}$ for all $i \geq 1$. The main result in this paper shows that an analogous theorem holds with reduced unmixed local ring and $I^{[l]}$ replacing analytically unramified semi-local ring and $\left(I^{l}\right)_{a}$, respectively, where $I^{[l]}$ is the intersection of certain primary ideals related to $I^{i}$. An application and a generalization are included.
\end{abstract}

1. Introduction. The theorem on analytically unramified semi-local rings in the abstract can be found in [15, Theorem 2], where it is actually shown that (1) and (2) are each equivalent to (3) holding for some open ideal $B\left(=\left(I^{m}\right)_{a}\right)$. And the equivalence of $(1)$ and (2) is also given in [14, Lemma 1 and Theorem 1.4]. I have always thought this theorem was rather beautiful, and it is also quite useful. For example, D. Rees developed it and then used it in [14] to characterize analytically unramified local rings as local rings $R$ all of whose finitely generated overrings have finite integral closures. (An overring of $R$ is a ring containing $R$ and contained in the total quotient ring of $R$.) This result verified a (modified) conjecture of $\mathrm{O}$. Zariski, [16].

There has recently been some renewed interest in large powers of ideals and their integral closures, and in some of the new results in this area the above theorem and the closely related Valuation Theorem of Rees, [12], have proved quite useful. (For example, see [10] and [11].) One of the main results in this paper, (13), shows that a similar and closely related theorem holds for ideals in reduced unmixed local rings. The ideals $I^{[i]}$ in the theorem are not as easily described as are the ideals $\left(I^{i}\right)_{a}$, but they are well defined ideals, and the theorem implies that if $I$ is any ideal in such a ring $R$ and $I^{\langle i\rangle}=\bigcap\left\{I^{i} R_{P} \cap R ; P\right.$ is a prime divisor of $\left.\left(I^{i}\right)_{a}\right\}$, then there exists an integer $n \geq 0$ such that $I^{\langle n+i\rangle} \subseteq I^{i}$ for all $i \geq 1$. (See (17).) For ideals $I$ of the principal class in reduced Cohen-Macaulay rings these theorems were previously known, since then $I^{i}=I^{[i]}=I^{\langle i\rangle}$ (see 
(18.2)), but the fact that these results hold for all ideals in such rings appears to be new.

Actually, it is shown in $\$ 2$ that the theorems hold somewhat more generally than for ideals in reduced locally unmixed Noetherian rings. (See (4), (5), (7), and the comment after (11).)

The results in $\$ 2$ are used in (20) to prove a result analogous to Rees' overring characterization of analytically unramified local rings.

In $\$ 3$ another result related to (2) above shows that for each regular ideal $I$ in a Noetherian ring there exists a sequence of ideals $I^{\{i\}}$ consisting of the intersection of certain primary ideals related to $I^{i}$ such that $I^{\{i\}}=I^{i}$ for all large $i$. ( $I$ is said to be regular in case $I$ contains a regular element.)

2. Powers of ideals. In order to prove the results concerning $I^{[i]}$, several definitions and preliminary remarks are needed. We begin by giving the definitions; a few comments on them are given in (2).

(1) Definitions. Throughout, $R$ is a Noetherian ring (commutative with identity) and $I$ is an ideal in $R$.

(1.1) The integral closure $I_{a}$ of $I$ in $R$ is the set of elements $x$ in $R$ that satisfy an equation of the form $X^{n}+i_{1} X^{n-1}+\cdots+i_{n}=0$, where $i_{j} \in I^{j}$.

(1.2) $A^{*}(I)=\operatorname{Ass}\left(R / I^{i}\right)$ for all large $i$, and $\hat{A}^{*}(I)=\operatorname{Ass}\left(R /\left(I^{i}\right)_{a}\right)$ for all large $i$.

(1.3) The Rees ring $\Re(R, I)$ of $R$ with respect to $I$ is the graded subring $R[u, t I]$ of $R[u, t]$, where $t$ is an indeterminate and $u=1 / t$.

(1.4) $\mathscr{T}(u \Re)$ is the ring $R[1 / u] \cap \Re_{(S)}\left(=R[u, t] \cap \Re_{(S)}\right)$, where $\Re=\Re(R, I)$ and $(S)$ is the set of regular elements in $S=\Re-$ $\cup\{p ; p$ is a height one prime divisor of $u \Re\}$.

(1.5) $I^{[i]}=u^{i} \mathcal{T} \cap R$, where $\mathcal{T}=\mathcal{T}(u \Re)$ with $\Re=\Re(R, I)$.

(1.6) A filtration on $R$ is a sequence of ideals $\left\{I_{i}\right\}_{i \geq 0}$ of $R$ such that $I_{0}=R, I_{i} \supseteq I_{i+1}$, and $I_{i} I_{j} \subseteq I_{i+j}$ for all $i$ and $j$. The filtration is an e.p.f. (essentially powers filtration) in case there exists an integer $k>0$ such that $I_{n}=\sum_{1}^{k} I_{n-i} I_{i}$ for all $n \geq 1$, where $I_{i}=R$ if $i \leq 0$.

(2) REMARK. (2.1) It is well known that $I_{a}$ is an ideal in $R$ such that $I \subseteq I_{a} \subseteq \operatorname{Rad} I$.

(2.2) It is known, [1], that the sets $\operatorname{Ass}\left(R / I^{i}\right)$ are equal for all large $i$, and a similar statement holds for the sets $\operatorname{Ass}\left(R /\left(I^{i}\right)_{a}\right)$, by [11], so $A^{*}(I)$ and $\hat{A}^{*}(I)$ are well defined (finite) sets of prime ideals in $R$.

(2.3) $\Re=\mathscr{R}(R, I)$ is a graded Noetherian ring, $u$ is a regular element in $\Re$, and $u^{i} \Re \cap I=I^{i}$ for all $i \geq 1$. 
(2.4) It is readily seen that $\mathcal{T}=\mathcal{T}(u \Re)$ is the set of elements which are expressible in the form $f_{i} / u^{i}$ for all large $i$, where $f_{l}$ is in every height one primary component of $u^{\imath} \mathscr{R}$, so $\mathcal{T}$ is a graded subring of $R[t, u]$, since $u^{i} \Re$ is a homogeneous ideal. Also, by [7, Lemma 5.15(4)], $u^{i} \widetilde{T}=u^{i} \Re{ }_{(S)} \cap$ $\widetilde{T}$, so $u^{i} \widetilde{T}$ is a finite intersection of height one primary ideals.

(2.5) Note that $I^{i}=u^{i} \Re \cap R \subseteq u^{i} \Im \cap R=I^{[l]}$, and it follows from this and the fact that $u^{i} \mathcal{T}=u^{i} \mathscr{R}_{(S)} \cap \mathcal{T}$ (see (2.4)) that $I^{[i]}$ is the intersection of some primary ideals related to $I^{i}$. However, even if $I$ is primary for the maximal ideal $M$ in a local domain $R$, it may not be true that $I^{[i]}=I^{i}$, since $I^{i}=\bigcap\left\{q_{i j} \cap R ; q_{i j}\right.$ is a primary component of $\left.u^{\imath} R\right\}$ may be properly contained in $I^{[i]}=\bigcap\left\{q_{i j} \cap R ; q_{i j}\right.$ is a height one primary component of $\left.u^{i} \Re\right\}$. (Using (13) below, a specific example of this can be constructed using [5, Example 2, pp. 203-205].)

(2.6) It is shown in $[9,(2.4 .3)]$ that a filtration $\left\{I_{i}\right\}$ is an e.p.f. if and only if there exists an integer $n \geq 1$ such that $I_{n+l}=I_{n} I_{l}$ for all $i \geq n$. Also, if $\left\{I_{l}\right\}$ is an e.p.f., then there exists an integer $m \geq 1$ such that $I_{m i}=\left(I_{m}\right)^{i}$ for $i \geq 1$, by [9, (2.4.4)]. (In regard to [9, (2.4.4)], it is not true that $I_{m i}=\left(I_{m}\right)^{i}$ for all large $m$ and for all $i \geq 1$.)

With the comment at the end of (2.6) in mind, it is still true that $I^{[m i]}=I^{[m] i}$ for all large $m$ and for all $i \geq 1$ for the filtration $\left\{I^{[i]}\right\}$ in reduced locally unmixed Noetherian rings, as is shown in (7) (together with (11)).

The definitions recalled in (1) have previously proved to be very useful concepts, so many results are known about them, beyond those mentioned in (2). Several of these will be needed below, so (3) contains a brief list of these additional results. (The references for (3.3) and (3.4) only prove the case for $R$ an integral domain, but that assumption was not essential for the proofs.)

(3) Remark. Let $I$ be an ideal in a Noetherian ring $R$, let $R=$ $\Re(R, I)$, and let $\mathcal{T}=\mathcal{T}(u \Re)$. Then the following statements hold:

(3.1) [7, Lemma 5.16]. If $R$ is a locally unmixed integral domain, then $\mathcal{T}$ is a finite $\Re$-algebra.

(3.2) [7, Corollary 4.9 and its proof]. If $R$ is local with maximal ideal $M$, if $I$ is generated by a system of parameters in $R$, and if $R$ is a subspace of $\mathcal{R}_{(M, u) \Re}$, where $\mathscr{R}=\mathfrak{R}(R, I)$, then $R$ is unmixed.

(3.3) [7, Lemma 5.15(5)]. $\mathcal{T}=\Re$ if and only if $u \Re$ has no imbedded prime divisors.

(3.4) [7, Lemma 5.15(9)]. If $S$ is a multiplicatively closed set in $\Re$, then $\sigma_{S}=\sigma_{(}\left(u \Re_{S}\right)$. 
We now begin to derive a few properties of the ideals $I^{[i]}$. The first of these gives several useful characterizations of when there exists an integer $n \geq 0$ such that $I^{[n+i]} \subseteq I^{i}$ for all $i \geq 1$. In the proof of (4), we use the notation $[H]_{i}=\left\{r \in R ; r t^{i} \in H\right\}$ for a homogeneous ideal $H$ in $\Re$. With this notation, it is clear that if $J$ is another homogeneous ideal in $R$, then $H \subseteq J$ if and only if $[H]_{i} \subseteq[J]_{i}$ for all $i(-\infty<i<\infty)$.

(4) Proposition. Let $I$ be an ideal in a Noetherian ring $R$, let $\Re=\Re(R, I)$, and let $\mathcal{T}=\mathcal{T}(u \Re)$. Then the following statements are equivalent:

(4.1) There exists an integer $n \geq 0$ such that $I^{[n+i]} \subseteq I^{i}$ for all $i \geq 1$.

(4.2) $\left\{I^{[i]}\right\}$ is an e.p.f. in $R$ and $\mathcal{T} \subseteq \Re^{\prime}$, the integral closure of $R$.

(4.3) $\mathcal{T}$ is a finite $\Re$-module.

(4.4) $u^{n+1} \mathcal{G} \cap \Re \subseteq u \Re$ for some $n \geq 0$.

(4.5) There exists an integer $n \geq 0$ such that $u^{n+i} \sigma \subseteq u^{i} \Re$ for all $i \geq 1$.

Proof. (4.2) $\Leftrightarrow$ (4.3), by [9, Theorem 2.7].

It is shown in [7, Lemma 5.15(10)] that (4.3) $\Leftrightarrow(4.4)$ when $R$ is an integral domain, and that assumption was not essential for the proof.

(4.3) $\Rightarrow(4.5)$, by the first statement in (2.4), and (4.5) implies that $I^{[n+i]}=u^{n+i} \mathcal{G} \cap R \subseteq u^{i} \Re \cap R=I^{i}$ for all $i \geq 1$, so (4.5) $\Rightarrow(4.1)$.

Finally, assume that (4.1) holds and let $u^{(i)}=u^{i} \mathcal{\top} \cap \Re$, so $u^{(i)}$ is the intersection of the height one primary components of $u^{i} \Re$, by the last statement in (2.4), hence $u^{(i)}$ is a homogeneous ideal. Now it is readily checked that $[u \Re]_{i}=I^{i+1}$ and $\left[u^{(n+1)}\right]_{i}=I^{i} \cap I^{[n+1+i]}$ for all $i$ (with the convention that $I^{i}=R=I^{[i]}$ for $i \leq 0$ ). Therefore (4.1) implies that $\left[u^{(n+1)}\right]_{i}=I^{i} \cap I^{[n+1+i]}=I^{[n+1+i]} \subseteq I^{i+1}=[u \Re]_{i}$ for all $i$, so $u^{n+1} \sigma \cap$ $\Re=u^{(n+1)} \subseteq u \Re$, and so (4.1) $\Rightarrow(4.4)$.

It should be noted that (4.3) $\Rightarrow(4.1)$ shows that if $I$ is an ideal in any Noetherian ring $R$ such that $\mathcal{T}=\mathscr{T}(u \Re)$ is a finite $R=\mathscr{R}(R, I)$-algebra, then there exists an integer $n \geq 0$ such that $I^{[n+i]} \subseteq I^{i}$ for all $i \geq 1$. It is shown in (11) that this holds for all ideals $I$ when $R$ is a reduced locally unmixed Noetherian ring.

Also concerning (4), if $\mathcal{T}$ is a finite $\mathcal{R}$-module, then $\left\{I^{[i]}\right\}$ is an e.p.f., by $(4.3) \Rightarrow(4.2)$, so there exists an integer $n \geq 1$ such that $I^{[n+i]}=I^{[i]} I^{[n]}$ for all $i \geq n$, by (2.6). (5) shows that we also have $I^{[n+i]}=I^{i} I^{[n]}$, when $\sigma$ is a finite $\Re$-module. The proof of (5) is essentially the same as that given in the cited reference, where it is shown that the analogous result for $\left(I^{n+i}\right)_{a}$ holds, so it will be omitted. 
(5) Corollary (cf. [15, Corollary, p. 37]). Let $R, I, \Re$, and $\mathcal{T}$ be as in (4) and assume that $\mathcal{T}$ is a finite $\mathcal{R}$-algebra. Then for all large integers $n$ and for all $i \geq 1$ it holds that $I^{[n+i]}=I^{i} I^{[n]}$.

(6) REMARK. It follows quite readily from (5) and (4.3) $\Leftrightarrow$ (4.1) together with their proofs that if there exists $n \geq 0$ such that $I^{[n+i]} \subseteq I^{i}$ for all $i \geq 1$, then $I^{[m+j]}=I^{[j]} I^{[m]}=I^{j} I^{[m]}$ for all $m \geq n$ and for all $j \geq 1$.

The next result is an analogue of (3) in the abstract.

(7) Proposition. Let I be an ideal in a locally unmixed Noetherian ring $R$ and assume that there exists an integer $n \geq 1$ such that $I^{[n+i]} \subseteq I^{i}$ for all $i \geq 1$. Fix $m \geq n$ and let $\Re=\Re\left(R, I^{[m]}\right)$ and $\mathcal{T}=\mathcal{T}(u \Re)$. Then $\mathcal{T}=\Re$ and $\left(I^{[m]}\right)^{[i]}=\left(I^{[m]}\right)^{i}=I^{[m i]}$ for all $i \geq 1$.

Proof. Let $\Re_{0}=\mathcal{R}(R, I), \mathscr{T}_{0}=\mathcal{T}\left(u \Re_{0}\right)$, and $\Re_{m}=R\left[u^{m}, t^{m} I^{[m]}\right]$, so $R \cong \Re_{m} \subseteq \mathcal{T}_{0}$. Now the hypothesis and (6) imply that $\mathcal{T}_{0}=$ $R\left[u, t I^{[1]}, \ldots, t^{m} I^{[m]}\right]$, and $\sigma_{0}$ is integrally dependent on $\mathcal{R}_{m}$, since $\left(t^{i} I^{[i]}\right)^{m}=t^{i m}\left(I^{[i]}\right)^{m} \subseteq t^{i m} I^{[i m]}=\left(t^{m} I^{[m]}\right)^{i}$ (since $\left\{I^{[i]}\right\}$ is an e.p.f. (by $(4.1) \Rightarrow(4.2))$ and since $I^{[i m]}=\left(I^{[m]}\right)^{i}$ (by (6))). Therefore let $p^{\prime}$ be a (height one) prime divisor of $u \mathcal{T}_{0}$ and let $z^{\prime}$ be a minimal prime ideal in $\mathcal{T}_{0}$ contained in $p^{\prime}$. Then $z=z^{\prime} \cap \Re_{m}$ is a minimal prime ideal contained in $p=p^{\prime} \cap \Re_{m}$ and $R /\left(z^{\prime} \cap R\right)$ satisfies the altitude formula, since $R$ is locally unmixed, so it follows from this and integral dependence that height $p / z=$ height $p^{\prime} / z^{\prime}=1$. Now, if $w$ is another minimal prime ideal contained in $p$, then using the altitude formula for $p / z$ and $p / w$ over $(p \cap R) /(z \cap R)$ and $(p \cap R) /(w \cap R)$, respectively, it follows that height $p=1$, since $R_{p \cap R}$ satisfies the first chain condition for prime ideals. Let $(S)$ be the set of regular elements in $S=\Re_{m}-\cup\{p ; p$ is a height one prime divisor of $\left.u \Re_{m}\right\}$. Then since the prime divisors of $u \mathscr{T}_{0}$ lie over the height one prime divisors of $u \Re_{m}$, it follows that $\mathscr{T}_{0}\left[1 / u^{m}\right] \cap$ $\mathcal{T}_{0(S)}=\mathscr{T}_{0}[1 / u] \cap \mathcal{T}_{0(S)}=\Re_{0}[1 / u] \cap \Re_{0\left(S^{\prime}\right)}=\mathcal{T}\left(u \Re_{0}\right)=\mathcal{T}_{0}$, where $\left(S^{\prime}\right)$ is the set of regular elements in $S^{\prime}=\Re_{0}-\cup\{q ; q$ is a height one prime divisor of $\left.u \Re_{0}\right\}$, so $\mathscr{T}_{m}=\Re_{m}\left[1 / u^{m}\right] \cap \Re_{m(S)} \subseteq \mathcal{T}_{0}$. Therefore since $\Re_{m}$ $\cong \Re$, it follows that $\left(I^{[m]}\right)^{[i]}=u^{i} \mathcal{T} \cap R=u^{m i} \sigma_{m} \cap R \subseteq u^{m i} \sigma_{0} \cap \Re_{m} \cap$ $R=u^{m i} \sigma_{0} \cap R=I^{[m i]}$, and the hypothesis implies that $I^{[m i]}=\left(I^{[m]}\right)^{i} \subseteq$ $\left(I^{[m]}\right)^{[i]}$. Therefore $\left(I^{[m]}\right)^{[i]}=I^{[m i]}=\left(I^{[m]}\right)^{i}$ for all $i \geq 1$, and so $\mathcal{T}=$ $R\left[u, t I^{[m]}, t^{2} I^{[2 m]}, \ldots\right]=R\left[u, t I^{[m]}\right]=\Re$.

It follows from (7) that $u \Re$ has no imbedded prime divisors, as is shown in the following corollary. 
(8) COROllary. Let I be an ideal in a locally unmixed Noetherian ring $R$ and assume that there exists an integer $n \geq 1$ such that $I^{[n+i]} \subseteq I^{i}$ for all $i \geq 1$. Then for all $m \geq n$ it holds that $u \Re\left(R, I^{[m]}\right)$ has no imbedded prime divisors.

Proof. $\mathscr{T}(u \Re)=\Re$, by (7), where $\Re=\mathscr{R}\left(R, I^{[m]}\right)$, and so the conclusion follows from (3.3).

(8) implies that the ideals $b R\left[I^{m} / b\right]$ have no imbedded prime divisors, as is shown in (9). (Actually, (9) holds for all non-nilpotent elements in $I^{m}$, but the notation becomes somewhat messy in proving this, so we content ourselves with the case when $b$ is regular.)

(9) COROllaRy. Let $R, I$, and $m \geq n$ be as in (8). Then for each regular element $b \in I^{m}$ such that $b R\left[I^{m} / b\right]$ is proper it holds that every prime divisor of $b R\left[I^{m} / b\right]$ has height one.

Proof. Let $A=R\left[I^{m} / b\right]$ and $\mathcal{R}=\Re\left(R, I^{m}\right)$. Then $A[t b, 1 / t b]=$ $\Re[1 / t b]$ and $b A[t b, 1 / t b]=u A[t b, 1 / t b]$. Therefore, since $t b$ is transcendental over $A$, it follows from (8) that $b A$ has no imbedded prime divisors.

As mentioned in (2.2), the sets $\operatorname{Ass}\left(R / I^{m}\right)$ are equal for all large $i$, and it is shown in [1] that they are not monotonically increasing. However, it is an open problem if $P \in A^{*}(I)$ and $P$ is a prime divisor of $I^{i}$ imply that $P$ is a prime divisor of $I^{j}$ for all $j \geq i$. (It is known that this holds for $\left(I^{i}\right)_{a}$ in place of $I^{i}$, by [10].) (10) shows that for the ideals $I^{[m]}$ the sets $\operatorname{Ass}\left(R /\left(I^{[m]}\right)^{i}\right)$ are increasing. In (10) and henceforth we use $I^{[m] i}$ to denote $\left(I^{[m]}\right)^{i}$, and likewise $I^{[m][i]}$ denotes $\left(I^{[m]}\right)^{[i]}$.

(10) Corollary. With $R, I$, and $m \geq n$ as in (8), if $P \in \operatorname{Spec} R$ is a prime divisor of $I^{[m] i}$ for some $i \geq 1$, then $P$ is a prime divisor of $I^{[m] j}$ for all $j \geq i$.

Proof. Let $J=I^{[m]}$, so $J^{i}: x R=P$ for some $x \in R$, by hypothesis. Let $\mathscr{R}=\mathscr{R}(R, J)$. Then $P \Re=\left(J^{i}: x R\right) \Re \subseteq J^{i} \Re: x \Re \subseteq u^{i} \Re: x \Re$, and $\left(u^{i} \mathscr{R}: x \mathscr{R}\right) \cap R=J^{i}: x R=P$, by $[17$, p. 220]. Therefore there exists a prime divisor $p$ of $u \Re$ such that $u^{i} \Re: x \Re \subseteq p$ and $p \cap R=P$. Now height $p=1$, by (8), so either height $P=0$ or height $P \geq 1$ and $t J \nsubseteq p$, by [10]. Now it may clearly be assumed that height $P \geq 1$, so there exists $b \in J$ such that $t b \notin p$. Therefore, for all $j \geq 1, u^{i+j} \mathscr{R}: x b^{j} \Re=u^{i} \Re$ : $x b^{j} t^{j} \Re \subseteq p$, so contracting to $R$ it follows that $J^{i+j}: x b^{j} R=P$. 
Recall that a ring $R$ is said to be reduced if $\operatorname{Rad} R=(0)$. With this terminology, we next show that if $R$ is reduced and locally unmixed, then the equivalent conditions in (4) hold for all ideals $I$ in $R$.

(11) Proposition. If $R$ is a reduced locally unmixed Noetherian ring, then for each ideal $I$ in $R$ there exists an integer $n \geq 0$ such that $I^{[n+i]} \subseteq I^{i}$ for all $i \geq 1$.

Proof. Let $I$ be an ideal in $R$ and let $\Re=\Re(R, I)$ and $\mathcal{T}=\mathscr{T}(u \Re)$. Let $T$ be the total quotient ring of $R$, so the prime divisors of zero in $\mathcal{T}$ and in $R$ are the ideals $z^{\prime}=z T \cap \mathcal{T}$ and $z^{*}=z T \cap \Re$ with $z$ prime divisor of zero in $R$. Now $R / z$ is locally unmixed, by [4, Proposition 3], and $R / z^{*} \cong \Re(R / z,(I+z) / z)$, by [13, Lemma 1.1$]$. To simplify notation we identify these two rings. Then $\mathscr{T}\left(u\left(\Re / z^{*}\right)\right)$ is a finite $\Re / z^{*}$-algebra, by (3.1). Also, if $P \in \operatorname{Spec} \Re$, then $\Re_{P} / z^{*} \Re_{P}$ is unmixed, by [4, Corollary, p. 61] (since $R / z$ is locally unmixed), and height $P / z^{*}=$ height $P$, as in the proof of (7), so $\Re_{P}$ satisfies the first chain condition for prime ideals. Therefore if $p$ is a height one prime divisor of $u\left(\Re / z^{*}\right)$, then there exists a prime ideal $P$ in $\Re$ such that $z^{*} \subset P$ and $P / z^{*}=p$, so necessarily $P$ is a height one prime divisor of $u \Re$. Thus, since $\Re_{(S)}$ is semi-local of altitude one, where $(S)$ is as in (1.5), it follows that

$$
\begin{aligned}
\mathcal{T} / z^{\prime} & =\left(R[t, u] \cap \Re_{(S)}\right) / z^{\prime} \subseteq(R / z)[t, u] \cap\left(R / z^{*}\right)_{\left(S+z^{*}\right) / z^{*}} \\
& =\mathcal{T}\left(u\left(\Re / z^{*}\right)\right) .
\end{aligned}
$$

Therefore, since $\operatorname{Rad} R=(0)$, it follows that $T(u \Re)$ is contained in a finite $R$-algebra, hence $\mathcal{T}(u \Re)$ is a finite $\mathcal{R}$-algebra, and so the conclusion follows from $(4.3) \Rightarrow(4.1)$.

I do not know to what extent (11) holds without the assumption that $\operatorname{Rad} R=(0)$. In particular, I do now know if $\cap\left\{R_{(p)} ; p\right.$ is a height one prime ideal in $R$ and $(p)$ is the set of regular elements in $R-p$ \} is a finite $R$-module for all unmixed local rings $(R, M)$. If this is always true, then this also holds for $\Re_{\Re}$ in place of $R$, where $R$ is unmixed, $I$ is an arbitrary ideal in $R, \Re=\Re(R, I)$, and $\Re=(u, M, t I) \Re$ (since $\Re_{\Re}$ is unmixed when $R$ is by [7, Theorem 4.1(2)(a)]), and it can then be readily shown that $\mathcal{T}(u \Re)$ is a finite $R$-module. In any case, whenever $R$ is locally unmixed and $\mathscr{T}(u \Re(R, I))$ is a finite $R(R, I)$-module the conclusions of all the results (4)-(20) hold for $I$, by (4).

Concerning (11), it should be noted that the conclusion is equivalent to each of the other four statements in (4). More will be said concerning this is (19) and the comments following (19). 
Most of the next two results are essentially corollaries to what has already been shown.

(12) THEOREM. The following statements are equivalent for a reduced Noetherian ring $R$ :

(12.1) $R$ is locally unmixed.

(12.2) For all ideals $I$ in $R$ there exists an integer $n \geq 0$ such that $I^{[n+i]} \subseteq I^{i}$ for all $i \geq 1$.

Moreover, if these statements hold, then for all ideals $I$ in $R$ and for all integers $m \geq n$ with $n$ as in (12.2), $B^{[i]}=B^{i}$ for all $i \geq 1$, where $B=I^{[m]}$.

Proof. (12.1) $\Rightarrow$ (12.2), by (11), and (12.2) implies the last statement in the theorem by (7). To complete the proof it suffices to show that (12.2) implies that $R_{M}$ is unmixed for all maximal ideals $M$ in $R$. For this, fix such an ideal $M$ and let $I$ be an ideal in $R$ such that $I_{M}$ is generated by a system of parameters. Let $\Re=\Re(R, I), \mathcal{T}=\mathscr{T}(u \Re)$, and $S=R-M$. Then $\Re_{S}=\Re\left(R_{M}, I R_{M}\right)$ and $\mathcal{T}_{S}=\sigma\left(u \Re_{S}\right)$, by (3.4). Now (12.2) implies

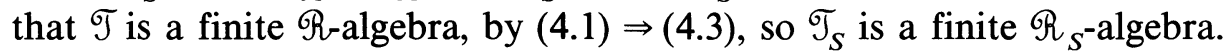
Also, $(M, u) \Re_{S}$ is the only minimal prime divisor of $u \Re_{S}$, by [7, Theorem 4.1(2)(b)] applied to $(M, u) R[u]$, so by the definition of $\mathcal{T}$ there exists a height one prime ideal $P$ in $\mathscr{T}_{S}$ that lies over $(M, u) \Re_{S}$ and then $u \mathscr{T}_{S}$ is $P$-primary. Therefore it follows from (4.3) $\Rightarrow$ (4.1) that $R_{M}$ is a subspace of $\left(\mathscr{T}_{S}\right)_{P}=\Re_{(M, u) \Re}$, hence $R_{M}$ is unmixed, by (3.2).

It should be noted that the proof of (12) showed that (12.2) $\Rightarrow(12.1)$ even when $\operatorname{Rad} R \neq(0)$. As already noted, I do not know to what extent the converse holds.

(13) THEOREM. The following statements are equivalent for a reduced local ring $R$ :

(13.1) $R$ is unmixed.

(13.2) There exists an ideal I generated by a system of parameters in $R$ and an integer $n \geq 0$ such that $I^{[n+i]} \subseteq I^{i}$ for all $i \geq 1$.

Moreover, if these statements hold and $I$ is any ideal in $R$, then the conclusion of (13.2) (with $n$ depending on I) holds for I and for all integers $m \geq n$ it holds that $B^{[i]}=B^{i}$ for all $i \geq 1$, where $B=I^{[m]}$.

Proof. (13.1) implies both (13.2) and the last statement, by (12), and the last part of the proof of (12) showed that (13.2) $\Rightarrow(13.1)$. 
On comparing (13.2) to (2) in the abstract it would seem that (13.2) should be stated "There exists an open ideal $I$ in $R$ and an integer $n \geq 0$ such that $I^{[n+i]} \subseteq I^{i}$ for all $i \geq 1$." Also, as noted in the introduction, $R$ is analytically unramified if and only if there exists an open ideal $I$ in $R$ such that $\left(I^{i}\right)_{a}=I^{i}$ for all $i \geq 1$, but (13) only shows that half of the analogous statement for unmixed and $I^{[i]}$ holds. (14) shows that the other half does not hold, and it is clear from this that the "open ideal" version of (13.2) also does not hold.

(14) EXAMPLE. There exists a local domain $R$ which is not unmixed such that $R$ has an open ideal $I$ such that $I^{[i]}=I^{i}$ for all $i \geq 1$. Namely, let $(R, M)$ be as in [5, Example 2, pp. 203-205] in the case $m=0$. Then $R$ is an analytically unramified local domain of altitude $r+1 \geq 2$ and there exists a height one maximal ideal in $R^{\prime}$, the integral closure of $R$, so $R$ is not unmixed, by [5, (34.7)]. However, since $R$ is analytically unramified, there exist open ideals $I$ such that $\left(I^{i}\right)_{a}=I^{i}$ for all $i \geq 1$. Fix such an ideal $I$ and let $\Re=\Re(R, I)$. Then essentially as in the proof that (4.1) $\Rightarrow(4.4)$ it follows that $u \Re=(u \Re)_{a}$, so $u \Re$ has no imbedded prime divisors, by [8, Corollary 2.11]. Therefore $\mathcal{T}(u \Re)=\Re$, by (3.3), and so $I^{[i]}=I^{i}$ for all $i \geq 1$, by (1.5) and (2.3).

(15) shows that (11) can be applied to more general rings than reduced locally unmixed ones.

(15) Corollary. Let $R$ be a reduced Noetherian ring and let $I$ be an ideal in $R$ such that $R_{P}$ is unmixed for all $P \in A^{*}(I)$. Then there exists an integer $n \geq 0$ such that $I^{[n+i]} \subseteq I^{i}$ for all large $i$.

Proof. Let $S=R-\cup\left\{P ; P \in A^{*}(I)\right\}$. Then $R_{S}$ is a reduced locally unmixed Noetherian ring, so there exists an integer $n \geq 0$ such that $\left(I_{S}\right)^{[n+i]} \subseteq\left(I_{S}\right)^{i}$ for all $i \geq 1$, by (11). Also, by considering $R \subseteq \mathcal{J} \subseteq \mathcal{J}_{S}$ and the ideal $u^{n+i} \mathcal{F}_{S}$, where $\mathcal{T}=\mathcal{T}(u \Re(R, I))$, it readily follows that $I^{[n+i]} \subseteq\left(I_{S}\right)^{[n+i]} \cap R$. Finally, $I^{i}=\left(I_{S}\right)^{i} \cap R$ for all large $i$, by the definition of $A^{*}(I)$, so the conclusion readily follows.

(16) REMARK. (16.1) If $I$ and $R$ are as in (15) and for all $i \geq 1$ and for all prime divisors $P$ of $I^{i}$ it holds that $R_{P}$ is unmixed, then it follows as in (15) (since there are only finitely many $P$ involved) that there exists an integer $n \geq 0$ such that $I^{[n+i]} \subseteq I^{i}$ for all $i \geq 1$. 
(16.2) A proof similar to that of (15) shows that if $I$ is an ideal in a Noetherian ring $R$ such that $R_{P}$ is analytically unramified for all $P \in$ $A^{*}(I)$, then there exists an integer $n \geq 0$ such that $\left(I^{n+i}\right)_{a} \subseteq I^{i}$ for all large $i$. And the result analogous to (16.1) also holds.

The next corollary and its related remark consider another ideal related to $I^{i}$.

(17) COROLlaRY. Let $I$ be an ideal in a reduced locally unmixed Noetherian ring $R$ and let $I^{\langle i\rangle}=\bigcap\left\{I^{i} R_{P} \cap R ; P \in \hat{A}^{*}(I)\right\}$. Then there exists an integer $n \geq 0$ such that $I^{\langle n+i\rangle} \subseteq I^{i}$ for all $i \geq 1$.

Proof. It is known, [10], that $\hat{A}^{*}(I)=\{p \cap R ; p$ is a prime divisor of $\left.(u \Re)_{a}\right\}$, where $\Re=\Re(R, I)$. Now $(u \Re)_{a}=u \Omega^{\prime} \cap \Re$, where $\Re^{\prime}$ is the integral closure of $\Re$, and all prime divisors of $u \Re{ }^{\prime}$ have height one, by [8, Proposition 2.13]. Therefore it follows as in the proof of (7) that all prime divisors of $(u \Re)_{a}$ have height one. Therefore $\hat{A}^{*}(I)=\{p \cap R ; p$ is a height one prime divisor of $u \Re\}$, so it follows immediately from the definitions that $I^{i} \subseteq I^{\langle i\rangle} \subseteq I^{[i]}$ for all $i \geq 1$. Now there exists an integer $n \geq 0$ such that $I^{[n+i]} \subseteq I^{i}$ for all $i \geq 1$, by (11), so $I^{\langle n+i\rangle} \subseteq I^{[n+i]} \subseteq I^{i}$.

(18) REMARK. Let $R$ be a reduced Noetherian domain.

(18.1) If $R$ is locally unmixed and $I$ is an ideal in $R$, then $I^{i} \subseteq I^{[i]} \subseteq$ $\left(I^{i}\right)_{a}$ for all $i \geq 1$.

(18.2) If $R$ is Cohen-Macaulay and $I$ is an ideal of the principal class (that is, $I$ can be generated by $h=$ height $I$ elements), then $I^{i}=I^{\langle i\rangle}=I^{[i]}$ $\subseteq\left(I^{i}\right)_{a}$ for all $i \geq 1$, where $I^{\langle i\rangle}$ is as in (17).

Proof. (18.1) Fix an ideal $I$ in $R$ and let $\Re=\Re(R, I)$ and $\mathcal{T}=\mathcal{T}(u \Re)$. Then $\mathcal{T}$ is a finite $R$-algebra, by (11) and (4.1) $\Rightarrow(4.3)$, so $\mathcal{T} \subseteq \Re^{\prime}$, the integral closure of $R$. Therefore $I^{i}=u^{i} \Re \cap R \subseteq u^{i} \mathcal{T} \cap R=I^{[i]} \subseteq u^{i} R^{\prime}$ $\cap R=\left(I^{i}\right)_{a}$.

(18.2) Fix an ideal $I$ of the principal class in $R$ and let $R=\Re(R, I)$ and $\mathcal{T}=\mathcal{T}(u \Re)$. Then $\Re$ is a Cohen-Macaulay ring, by [6, Theorem 3.1], and $u$ is an $\Re$-sequence, so all prime divisors of $u \Re$ have height one, by [18, Theorem 2, p. 397]. Therefore $\mathcal{T}=\Re$, by (3.3), and so $I^{i}=I^{[i]}$, and $I^{[i]} \subseteq\left(I^{i}\right)_{a}$, by (18.1). Finally, $I^{i} \subseteq I^{\langle i\rangle} \subseteq I^{[i]}$, by the proof of (17).

This section will be closed with the following remark and related theorem. 
(19) REMARK. It follows immediately from (12) and (4.1) $\Rightarrow$ (4.3) that a reduced Noetherian ring $R$ is locally unmixed if and only if, for all ideals $I$ in $R, \mathcal{T}(u \Re)$ is a finite $R$-module, where $R=\mathscr{R}(R, I)$.

As mentioned in the introduction, Rees used the equivalence of (1) and (2) in the abstract to characterize analytically unramified local rings in terms of finitely generated overrings in [14]. The results in this paper, especially (12) and (13), can be used to obtain the following characterization of an unmixed local domain which is analogous to Rees' theorem and is also closely related to (19). (The characterization in (20) can be extended to reduced locally unmixed Noetherian rings. However, my only proof for this more general case involves a number of ideas which have not been previously considered in this paper, so we restrict attention to the case of a local domain with an infinite residue field.) In (20), $\mathscr{T}(b A)$ is defined the same way that $\mathcal{T}(u \Re)$ was, namely, $\mathscr{T}(b A)=A[1 / b] \cap A_{S}$, where $S=A-\cup\{p ; p$ is a height one prime divisor of $b A\}$, and results similar to (2.4) hold for $\mathcal{T}(b A)$, by [7, Lemma 5.15]. Also for (20), recall that an element $b$ in an ideal $I$ in a Noetherian domain $R$ is a superficial element for $I$ in case $I^{n+1}: b R=I^{n}$ for all large $n$. (See [18, (3), p. 285 and (5), p. 273].)

(20) TheOREM. Let $(R, M)$ be a local domain such that $R / M$ is infinite. Then $R$ is locally unmixed if and only if $\mathcal{T}(b A)$ is a finite A-module for all finitely generated overrings $A$ of $R$ and for all nonzero elements $b$ in $A$.

Proof. Assume first that $R$ is unmixed and fix $A$ and $b$ as in the theorem. Then $A$ is locally unmixed, by [4, Corollary, p. 61] and $\operatorname{Rad} A=$ (0), so $\mathcal{T}=\mathcal{T}(u \Re)$ is a finite $\Re$-algebra, by (19), where $\Re=\Re(A, b A)$. Thus by (4.3) $\Rightarrow(4.1)$ there exists $n$ such that $(b A)^{[n+i]} \subseteq b^{i} A$ for all $i \geq 1$, so let $B=A\left[(b A)^{[1]} / b, \ldots,(b A)^{[n]} / b^{n}\right]$. Then $\mathcal{T}[1 / t b]=B[t b, 1 / t b]$ is a finite $R[1 / t b]=A[t b, 1 / t b]$-module, so it follows that $B$ is a finite $A$-module and also that every prime divisor of $b B$ has height one, since this holds for $b \mathscr{T}[1 / t b]=u \mathscr{T}[1 / t b]$, by (2.4). Therefore $\mathscr{T}(b A) \subseteq \mathcal{T}(b B)$ $=B$, by (2.4) and since height one prime ideals in $B$ lie over height one prime ideals in $A$ (since $A$ satisfies the altitude formula), so $\mathscr{T}(b A)$ is a finite $A$-module.

For the converse, let $I$ be a nonzero ideal in $R$ and let $b$ be a superficial element for $I$. (Such elements exist, since $R / M$ is infinite.) Let $\Re=\Re(R, I), \mathcal{T}=\mathcal{T}(u \Re), A=R[I / b]$, and $B=\mathcal{T}(b A)$, so $B$ is a finite $A$-module and it readily follows that $b^{i} A \cap R=I^{i}$ for all large $i$, since $b$ is 
superficial for $I$. Now $C=B[t b, 1 / t b]$ is a finite $\mathscr{R}[1 / t b]=A[t b, 1 / t b]$ module and $b C=u C$ has no imbedded prime divisors, since $b B$ has none. Also, height one prime divisors of $b C$ lie over height one prime divisors of $b A[t b, 1 / t b]=u \Re[1 / t b]$, so $\mathscr{T}(u \Re[1 / t b])=\mathcal{T}[1 / t b] \subseteq C$ (3.4). Since $b$ is superficial for $I, t b$ is not in any relevant prime divisor of $u \Re$, by [2, Theorem 3], so $t b$ is not in any (height one) prime divisor of $u \mathscr{T}$. Therefore, since $B$ is a finite $A$-module, there exists $n \geq 0$ such that for all large $i$ it holds that $I^{[n+i]}=u^{n+i} \sigma \cap R=u^{n+i} \sigma[1 / t b] \cap R \subseteq u^{n+i} C \cap$ $R=b^{n+i} C \cap R=b^{n+i} B \cap R \subseteq b^{i} A \cap R=I^{i}$, and it readily follows from this and (12) that $R$ is unmixed.

3. A generalization. In this brief section it is shown that a result analogous to $\left(I^{n+i}\right)_{a} \subseteq I^{i}$ and to $I^{[n+i]} \subseteq I^{i}$ holds for all regular ideals $I$ in all Noetherian rings.

(21) THEOREM. Let I be a regular ideal in a Noetherian ring $R$, let $\Re=\Re(R, I)$, and let $\mathscr{Q}=\Re_{(S)} \cap R[t, u]$, where $(S)$ is the set of regular elements in $S=\Re-\cup\{P ; P$ is a relevant prime divisor of $u \Re\}$. For $i \geq 1$ let $I^{\{i\}}=u^{i} \cup \cap R$. Then $I^{\{i\}}=I^{i}$ for all large $i$.

Proof. Viewing $\mathscr{U}$ as a transformation $\mathcal{U}(u \Re)$ of a graded ring $\Re$, it is readily seen that $\mathscr{Q}\left(u \Re_{U}\right)=\mathcal{Q}_{U}$, for all multiplicatively closed sets $U$ in $R$, and that $\mathscr{Q}(u \Re[X])=\mathscr{Q}[X]$, where $X$ is an indeterminate, so it may be assumed that $R$ is local with an infinite residue field. Therefore let $b$ be a superficial element for $I$. Then $t b$ is not in any relevant prime divisor of $u \Re$, by [2, Theorem 3], and since $I$ is regular it may be assumed that $b$ is regular. Therefore $I^{n+1}: b R=I^{n}$ for all large $n$, by [18, (3), p. 285 and (5), p. 273], so it readily follows that $b^{n} R[I / b] \cap R=I^{n}$ for all large $n$. Also, since $t b$ is in a prime divisor $P$ of $u \Re$ if and only if $P$ is irrelevant, it follows that there exists a one-to-one correspondence between the prime divisors of $(u)$ in the rings $\Re_{(S)}, \Re[1 / t b]_{(S)}, \Re[1 / t b]$, and $\mathcal{U}_{(S)}$. Let $A=R[I / b]$, so $\Re[1 / t b]=A[t b, 1 / t b]$ and $u \Re[1 / t b]=b \Re[1 / t b]$. Therefore it follows that $I^{\{i\}}=u^{i} \mathscr{Q} \cap R=u^{i} \Re_{(S)} \cap R=u^{i} \Re[1 / t b] \cap$ $R=b^{i} \Re[1 / t b] \cap R=b^{i} A \cap R=I^{i}$ for all large $i$.

It follows as in (2.4) that $\mathcal{U}$ is a graded subring of $R[t, u]$, so it follows immediately from (21) that $\mathcal{Q}$ is a finite $\mathcal{R}$-algebra.

The following corollary of (21) is known, [3, Corollary 17], but the proof of (21) gives a nice alternate proof of one containment. It should be 
noted that the conclusion of (21) does not immediately follow from (22), since it is not clear that $u^{i} \mathscr{Q} \cap R=\bigcap\left\{I^{i} R_{p} ; p \in A^{*}(I)\right\}$ for all large $i$ (since possibly some irrelevant prime divisor of $u^{i} \Re$ lies over some $\left.p \in A^{*}(I)\right)$.

(22) CoRollary. With $R, I$, and $\Re$ as in (21), $A^{*}(I)=\{P \cap R ; P$ is a relevant prime divisor of $u \Re\}$.

Proof. It follows immediately from (21) that $A^{*}(I) \subseteq\{P \cap R ; P$ is a relevant prime divisor of $u \Re\}$. For the opposite inclusion, let $P$ be a relevant prime divisor of $u \Re$, so $u \Re: c t^{k} \Re=P$ for some homogeneous element $c t^{k}$ in $\Re$. Since $P$ is relevant there exists $d t \in \Re-P$, so $u \Re$ : $c t^{k} d^{n} t^{n} \Re=P$ for all $n \geq 0$. Therefore $u^{n+k+1} \Re: c d^{n} \Re=P$, and contracting to $R$ it follows that $I^{n+k+1}: c d^{n} R=P \cap R$ for all $n \geq 0$, so $P \cap R \in A^{*}(I)$.

\section{REFERENCES}

[1] M. Brodmann, Asymptotic stability of $\operatorname{Ass}\left(M / I^{n} M\right)$, Proc. Amer. Math. Soc., 74 (1979), 16-18.

[2] D. Kirby, A note on superficial elements of an ideal in a local ring, Quart. J. Math. Oxford Ser., 14 (1963), 21-28.

[3] S. McAdam and P. Eakin, The asymptotic Ass, J. Algebra, 61 (1979), 71-81.

[4] M. Nagata, On the chain problem of prime ideals, Nagoya Math. J., 10 (1956), 51-64.

[5] __ Local Rings, Interscience Tracts 13, Interscience, New York, 1962.

[6] L. J. Ratliff, Jr., Two notes on locally Macaulay rings, Trans. Amer. Math. Soc., 119 (1965), 278-284.

[7] _ On quasi-unmixed local domains, the altitude formula, and the chain condition for prime ideals (II), Amer. J. Math., 92 (1970), 99-144.

[8] - On prime divisors of the integral closure of a principal ideal, J. Reine Angew. Math., 255 (1972), 210-220.

[9] __ Notes on essentially powers filtrations, Michigan Math. J., 26 (1979), 313-324.

[10] __ On asymptotic prime divisors, Pacific J. Math., (to appear).

[11] _ Independent elements and asymptotic sequences, J. Algebra, (to appear).

[12] D. Rees, Valuations associated with a local ring (II), J. London Math. Soc., 31 (1956), 228-235.

[13] __ A note on form rings and ideals, Mathematika, 4 (1957), 51-60.

[14] _ A note on analytically unramified local rings, J. London Math. Soc., 36 (1961), 24-28.

[15] M. Sakuma and H. Okuyama, On a criterion for analytically unramification of a local ring, J. Gakugei, Tokushima Univ., 15 (1966), 36-38.

[16] O. Zariski, Analytical irreducibility of normal varieties, Annals of Math., 49 (1948), 352-361. 
[17] O. Zariski and P. Samuel, Commutative Algebra, Vol. I, Van Nostrand, New York, 1958.

[18] , Commutative Algebra, Vol. II, Van Nostrand, New York, 1960.

Received September 18, 1981 and in revised form June 4, 1982. Research on this paper was suppoorted in part by the National Science Foundation, Grant MCS8001597-A01.

UNIVERSITY OF CALIFORNIA

RIVERSIDE, CA 92521 


\title{
PACIFIC JOURNAL OF MATHEMATICS \\ EDITORS
}

\author{
DONALD BABBITT (Managing Editor) \\ University of California \\ Los Angeles, CA 90024 \\ Hugo Rossi \\ University of Utah \\ Salt Lake City, UT 84112 \\ C. C. Moore and Arthur Ogus \\ University of California \\ Berkeley, CA 94720
}

\author{
J. DugundiI \\ Department of Mathematics \\ University of Southern California \\ Los Angeles, CA 90089-1113
}

R. FINN and H. SAMELSON

Stanford University

Stanford, CA 94305

\begin{tabular}{lllll}
\multicolumn{3}{c}{ ASSOCIATE EDITORS } \\
R. ARENS & $\begin{array}{lll}\text { E. F. BeCKendach } \\
(1906-1982)\end{array}$ & B. H. NeumanN & F. Wolf & K. Yoshida
\end{tabular}

\begin{tabular}{ll}
\multicolumn{2}{c}{ SUPPORTING INSTITUTIONS } \\
UNIVERSITY OF ARIZONA & UNIVERSITY OF OREGON \\
UNIVERSITY OF BRITISH COLUMBIA & UNIVERSITY OF SOUTHERN CALIFORNIA \\
CALIFORNIA INSTITUTE OF TECHNOLOGY & STANFORD UNIVERSITY \\
UNIVERSITY OF CALIFORNIA & UNIVERSITY OF HAWAII \\
MONTANA STATE UNIVERSITY & UNIVERSITY OF TOKYO \\
UNIVERSITY OF NEVADA, RENO & UNIVERSITY OF UTAH \\
NEW MEXICO STATE UNIVERSITY & WASHINGTON STATE UNIVERSITY \\
OREGON STATE UNIVERSITY & UNIVERSITY OF WASHINGTON
\end{tabular}

The Supporting Institutions listed above contribute to the cost of publication of this Journal, but they are not owners or publishers and have no responsibility for its content or policies.

Mathematical papers intended for publication in the Pacific Journal of Mathematics should be in typed form or offset-reproduced (not dittoed), double spaced with large margins. Please do not use built up fractions in the text of the manuscript. However, you may use them in the displayed equations. Underline Greek letters in red, German in green, and script in blue. The first paragraph must be capable of being used separately as a synopsis of the entire paper. In particular it should contain no bibliographic references. Please propose a heading for the odd numbered pages of less than 35 characters. Manuscripts, in triplicate, may be sent to any one of the editors. Please classify according to the scheme of Math. Reviews, Index to Vol. 39. Supply name and address of author to whom proofs should be sent. All other communications should be addressed to the managing editor, or Elaine Barth, University of California, Los Angeles, California 90024.

There are page-charges associated with articles appearing in the Pacific Journal of Mathematics. These charges are expected to be paid by the author's University, Government Agency or Company. If the author or authors do not have access to such Institutional support these charges are waived. Single authors will receive 50 free reprints; joint authors will receive a total of 100 free reprints. Additional copies may be obtained at cost in multiples of 50 .

The Pacific Journal of Mathematics is issued monthly as of January 1966. Regular subscription rate: $\$ 132.00$ a year (6 Vol., 12 issues). Special rate: $\$ 66.00$ a year to individual members of supporting institutions.

Subscriptions, orders for numbers issued in the last three calendar years, and changes of address should be sent to Pacific Journal of Mathematics, P.O. Box 969, Carmel Valley, CA 93924, U.S.A. Old back numbers obtainable from Kraus Periodicals Co., Route 100, Millwood, NY 10546.

The Pacific Journal of Mathematics ISSN 0030-8730 is published monthly by the Pacific Journal of Mathematics at P.O. Box 969, Carmel Valley, CA 93924. Application to mail at Second-class postage rates is pending at Carmel Valley, California, and additional mailing offices. Postmaster: Send address changes to Pacific Journal of Mathematics, P. O. Box 969, Carmel Valley, CA 93924.

PUBLISHED BY PACIFIC JOURNAL OF MATHEMATICS, A NON-PROFIT CORPORATION

Copyright $\odot 1983$ by Pacific Journal of Mathematics 


\section{Pacific Journal of Mathematics}

Vol. 107, No. $2 \quad$ February, 1983

Driss Abouabdillah, Topologies de corps $A$ linéaires $\ldots \ldots \ldots \ldots \ldots . \ldots 257$

Patrick Robert Ahern, On the behavior near a torus of functions

holomorphic in the ball $\ldots \ldots \ldots \ldots \ldots \ldots \ldots \ldots \ldots \ldots \ldots \ldots \ldots . \ldots 267$

Donald Werner Anderson, There are no phantom cohomology operations

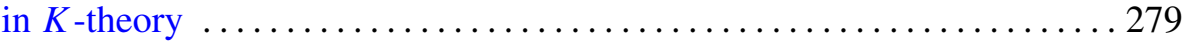

Peter Bloomfield, Nicolas P. Jewell and Eric Hayashi, Characterizations of

completely nondeterministic stochastic processes . ............. 307

Sydney Dennis Bulman-Fleming and K. McDowell, Absolutely flat

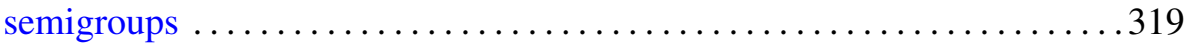

C. Debiève, On a Radon-Nikodým problem for vector-valued measures . . . 335

Dragomir Z. Djokovic, Products of positive reflections in real orthogonal

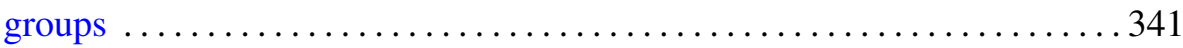

Thomas Farmer, The dual of the nilradical of the parabolic subgroups of

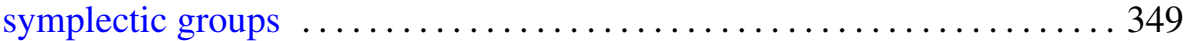

Gary R. Greenfield, Uniform distribution in subgroups of the Brauer group of an algebraic number field $\ldots \ldots \ldots \ldots \ldots \ldots \ldots \ldots \ldots \ldots \ldots . \ldots \ldots$

Paul Daniel Hill, When $\operatorname{Tor}(A, B)$ is a direct sum of cyclic groups $\ldots \ldots \ldots 383$

Hiroshi Maehara, Regular embeddings of a graph $\ldots \ldots \ldots \ldots \ldots \ldots \ldots 3$

Nikolaos S. Papageorgiou, Nonsmooth analysis on partially ordered vector spaces. I. Convex case . .............................4 403

Louis Jackson Ratliff, Jr., Powers of ideals in locally unmixed Noetherian

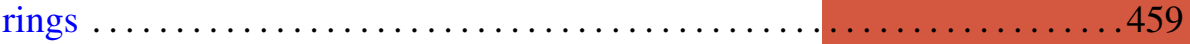

F. Dennis Sentilles and Robert Francis Wheeler, Pettis integration via the Stonian transform .......................... 